\title{
Comparison theorem between Fourier transform and Fourier transform with compact support
}

\author{
Christine Huyghe
}

December 4, 2012

\begin{abstract}
We prove a comparison theorem between Fourier transform without support and and Fourier transform with compact support in the context of arithmetic $\mathcal{D}$-modules.
\end{abstract}

\section{Résumé}

Nous démontrons un théorème de comparaison entre la transformation de Fourier à support compact et la transformation de Fourier sans support, pour les $\mathcal{D}$-modules arithmétiques.

\section{Contents}

1 Preliminaries

2 Fourier transforms

3 Comparison theorem

\section{Introduction}

Let $p$ be a prime number, $V$ a discrete valuation ring of inequal characteristics $(0, p)$, containing an element $\pi$, the $\pi$ of Dwork, satisfying the equation $\pi^{p-1}=-p, \mathcal{S}=\operatorname{Spf}(V)$ the formal spectrum of $V, X$ the formal affine line over $\mathcal{S}, X^{\vee}$ the dual affine line. Let us introduce $y$ and $y^{\vee}$ two copies of the formal projective line over $\mathcal{S}$ compactifying $x$ and $x^{\vee}$ and denote $\infty y$ (resp. $\infty y v)$ the complementary divisors. In [Ber96] Berthelot constructed 
sheaves of arithmetic differential operators with overconvergent singularities along a divisor

(e.g. $\left.\mathcal{D}_{y}^{\dagger}\left(\infty_{y}\right)\right)$ in our situation). For several reasons these sheaves have to be thought as sheaves over the open subset which is the complementary of the considered divisor, thus, in our case, over the formal affine line. In Huy95a one constructed the Fourier transform of $\mathcal{D}_{y}^{\dagger}(\infty y)$-modules, using the Dwork exponential module as kernel and we checked the compatibility with the so-called naïve Fourier transform. The aim of this article is to define the compact support Fourier transform in dimension 1 and to prove that it coincides with the Fourier transform without compact support introduced in loc. cit.. This result, already known in the complex case, is thus the $p$-adic analogous of the comparison theorem 2.4.1 of Katz-Laumon for the Fourier transform of $l$-adic sheaves ([KL85]).

This result was originally part from the unpublished part 4.4 of Huy95a, where it is proven in dimension $N$. Recently Abe and Marmora ([AM11) gave a proof of the product formula for $p$-adic epsilon factors, using this comparison theorem between Fourier and Fourier with compact support for curves and making necessary its publication, at least in the case of dimension 1 . The extra work in dimension $N$ consists into proving a generalization of the division lemma 2.4.1 and to deal with longer complexes of length $N+1$.

Compared with the original proof, our redaction takes into account recent progress in the understanding of the sheaves of Berthelot, which allows us to simplify the exposition of the proof. The main difference with the complex case, which makes things technically heavier, is that we have to work with sheaves with overconvergent coefficients at infinity over the formal projective line, instead of working directly with sheaves over the affine formal line. We surmount this difficulty thanks to the equivalence of categories given in 1.5.1. One key point of the proof is the fact that the Fourier transform with compact support preserves coherence (2.4.2), which relies on a division lemma (2.4.1). Then, to establish the comparison theorem, by (1.5.1), we may reduce the statement to the case of the sheaf $\left.\mathcal{D}_{y}^{\dagger}(\infty y)\right)$ for which we can give an explicit computation.

\section{Preliminaries}

\section{$1.1 \quad$ Notations}

Denote $K$ the fraction field of $V$. For $l \in \mathbf{Z},|l|$ is the usual archimedean absolute value of $l$. The $p$-adic valuation of an element $a$ of a $p$-adically separated ring is $v_{p}(a)$. For $a \in K$, 
let us state

$$
|a|_{p}=p^{-v_{p}(a)}
$$

If $\mathcal{F}$ is a sheaf of abelian groups over a topological space we denote $\mathcal{F}_{\mathbf{Q}}=\mathbf{Q} \otimes_{\mathbf{Z}} \mathcal{F}$.

The product $z=y \times y^{\vee}=\mathbb{P}_{\mathcal{S}}^{1} \times \mathbb{P}_{\mathcal{S}}^{1}$ is endowed with the ample divisor $\infty=\infty y \times$ $\mathbb{P}_{\mathcal{S}}^{1} \cup \mathbb{P}_{\mathcal{S}}^{1} \times \infty y$.

When needed, we will use $\left[u_{0}, u_{1}\right]$ and $\left[v_{0}, v_{1}\right]$ as homogeneous coordinates over the two copies of $\mathbb{P}_{\mathcal{S}}^{1}, u_{0}=0$ and $v_{0}=0$ are equations of the infinite divisor over each copy of $\mathbb{P}_{\mathcal{S}}^{1}$, $x=u_{1} / u_{0}$ and $y=v_{1} / v_{0}$ will be coordinates over the affine plane complementary of the $\infty$ divisor over $z$. These two coordinates $x$ and $y$ over $X=\mathbb{A}_{\S}^{1}$ and $X^{\vee}=\mathbb{A}_{\S}^{1}$ should be considered as dual to each other.

\subsection{Sheaves of arithmetic differential operators}

Let $\mathcal{V}$ be a smooth formal scheme over $\mathcal{S}$, endowed with a relative divisor $D$ (meaning that $D$ induces a divisor of the special fiber), and $\mathcal{U}=\mathcal{V} \backslash D$. Then the direct image by specialization of the constant overconvergent $F$-isocrystal over $U$, the special fiber of $\mathcal{U}$, is a sheaf over $\mathcal{V}$ denoted by $\mathcal{O}_{\mathcal{V}}\left({ }^{\dagger} D\right)$ (4.4 of [Ber96]). If $\mathcal{V}$ is affine and if $f$ is an equation of $D$ over $\mathcal{V}$, we have the following description

$$
\Gamma\left(\mathcal{V}, \mathcal{O}_{\mathcal{V}}\left({ }^{\dagger} D\right)\right)=\left\{\sum_{l \in \mathbf{N}} \frac{a_{l}}{f^{l}}, a_{l} \in \Gamma\left(\mathcal{V}, \mathcal{O}_{\mathcal{V}, \mathbf{Q}}\right) \mid \text { and } \exists C^{\prime}>0 \mid v_{p}\left(a_{l}\right)-\frac{l}{C^{\prime}} \rightarrow+\infty \text { if } l \rightarrow+\infty\right\} .
$$

Consider the ring $\mathcal{D}_{\mathcal{V}}^{\dagger}$ of arithmetic differential operators over $\mathcal{V}$. Suppose that $x_{1}, \ldots, x_{n}$ are coordinates on $\mathcal{V}$, denote $\partial_{x_{i}}$ the corresponding derivations for $i \in\{1, \ldots, n\}$, and

$$
\partial_{x_{i}}^{\left[k_{i}\right]}=\frac{\partial_{x_{i}}^{k_{i}}}{k_{i} !} \text {, and } \underline{\partial}^{[\underline{k}]}=\partial_{x_{1}}^{\left[k_{1}\right]} \cdots \partial_{x_{n}}^{\left[k_{n}\right]} \text {, }
$$

then we have the following description

$$
\Gamma\left(\mathcal{V}, \mathcal{D}_{\mathcal{V}}^{\dagger}\right)=\left\{\sum_{\underline{k} \in \mathbf{N}^{n}} a_{\underline{k}} \underline{\partial}^{[\underline{k}]}, a_{\underline{k}} \in \Gamma\left(\mathcal{V}, \mathcal{O}_{\mathcal{V}, \mathbf{Q}}\right) \text { and } \exists C>0 \mid v_{p}\left(a_{\underline{k}}\right)-\frac{|\underline{k}|}{C} \rightarrow+\infty \text { if }|\underline{k}| \rightarrow+\infty\right\} \text {. }
$$

In 4.2 of [Ber96], Berthelot introduces also rings of differential operators with overconvergent singularities, which are sheaves of $\mathcal{O}_{\mathcal{V}}\left({ }^{\dagger} D\right)$-modules. Suppose that $x_{1}, \ldots, x_{n}$ are coordinates on $\mathcal{V}$ and that the divisor $D$ is defined by the equation $f=0$ on $\mathcal{V}$, then we 
have the following description

$$
\begin{array}{r}
\Gamma\left(\mathcal{V}, \mathcal{D}_{\mathcal{V}}^{\dagger}(D)\right)=\left\{\sum_{l \in \mathbf{N}, \underline{k} \in \mathbf{N}^{n}} \frac{a_{l, \underline{k}}}{f^{l}} \underline{\underline{k}}^{[\underline{k}]}, a_{l, \underline{k}} \in \Gamma\left(\mathcal{V}, \mathcal{O}_{\mathcal{V}, \mathbf{Q}}\right)\right. \\
\text { and } \left.\exists C>0 \mid v_{p}\left(a_{l, \underline{k}}\right)-\frac{l+|\underline{k}|}{C} \rightarrow+\infty \text { if } l+|\underline{k}| \rightarrow+\infty\right\} .
\end{array}
$$

All these sheaves are weakly complete, in the sense that they are inductive limit of sheaves of $p$-adic complete rings as described in the following subsection. Let us stress here upon the fact that these sheaves are always sheaves of $K$ vector spaces (event if there is no $\mathbf{Q}$ in their notation). We made this convention to avoid too heavy notations. Note also that this won't be the case with the sheaves that we will introduce in the following subsections.

\subsection{Sheaves of arithmetic differential operators with overconvergent co- efficients}

In this subsection, we use notations of 1.2 . Let us fix $m \in \mathbf{N}$, elements $x_{1}, \ldots, x_{n} \in \Gamma\left(\mathcal{V}, \mathcal{O}_{\mathcal{V}}\right)$ which are coordinates on $\mathcal{V}$ and $f \in \Gamma\left(\mathcal{V}, \mathcal{O}_{\mathcal{V}}\right)$ such that $D \bigcap \mathcal{V}=V(f)$.

Let us introduce here some coefficients. First we define the application $\nu_{m}: \mathbf{Z} \rightarrow \mathbf{N}$ by the following way. If $k<0$, we set $\nu_{m}(k)=0$. Let $k \in \mathbf{N}$, and $q$ and $r$ be the quotient and the remainder of the division of $k$ by $p^{m+1}$. If $r=0$, we set $\nu_{m}(k)=q$, otherwise we set $\nu_{m}(k)=q+1$. We extend this application to $\mathbf{Z}^{r}$, by $\nu_{m}\left(\left(k_{1}, \ldots, k_{r}\right)\right)=\nu_{m}\left(k_{1}\right)+\cdots+\nu_{m}\left(k_{r}\right)$.

We denote also $q_{k}^{(m)}$ the quotient of the division of a positive integer $k$ by $p^{m}$, and

$$
\underline{\partial}^{\langle\underline{k}\rangle_{(m)}}=q_{k_{1}}^{(m)} ! \cdots q_{k_{n}}^{(m)} ! \partial_{x_{1}}^{\left[k_{1}\right]} \cdots \partial_{x_{n}}^{\left[k_{n}\right]} .
$$

If the choice of $m$ is clear, we will omit it in the notation.

Berthelot defines a sheaf $\widehat{\mathcal{B}}_{\mathcal{V}}^{(m)}$ by setting locally

$\Gamma\left(\mathcal{V}, \widehat{\mathcal{B}}_{\mathcal{V}}^{(m)}\right)=\left\{\sum_{l} \frac{a_{l}}{f^{l}}, a_{l} \in \Gamma\left(\mathcal{V}, \mathcal{O}_{\mathcal{V}}\right) \mid v_{p}\left(a_{l}\right) \geq \nu_{m}(l)\right.$ and $v_{p}\left(a_{l}\right)-\nu_{m}(l) \rightarrow+\infty$ if $\left.l \rightarrow+\infty\right\}$

Then there are canonical injective morphisms $\widehat{\mathcal{B}}_{\mathcal{V}}^{(m)} \subset \widehat{\mathcal{B}}_{\mathcal{V}}^{(m+1)}$ and

$$
\mathcal{O}_{\mathcal{V}}\left({ }^{\dagger} D\right)=\underset{m}{\lim } \widehat{\mathcal{B}}_{\mathcal{V}, \mathbf{Q}}^{(m)}
$$


Berthelot defines also sheaves of rings differential operators $\mathcal{D}_{\mathcal{V}}^{(m)}(D)$ and their $p$-adic completion $\widehat{\mathcal{D}}_{\mathcal{V}}^{(m)}(D)$ over $\mathcal{V}$ by

$$
\Gamma\left(\mathcal{V}, \mathcal{D}_{\mathcal{V}}^{(m)}(D)\right)=\left\{\sum_{l \in \mathbf{N}, \underline{k} \in \mathbf{N}^{n}} \frac{a_{l, \underline{k}}}{f^{l}} \underline{\partial}^{\langle\underline{k}\rangle_{(m)}}, a_{l, \underline{k}} \in \Gamma\left(\mathcal{V}, \mathcal{O}_{\mathcal{V}}\right) \mid v_{p}\left(a_{l, \underline{k}}\right) \geq \nu_{m}(l)\right\},
$$

where the sums are finite and

$$
\begin{array}{r}
\Gamma\left(\mathcal{V}, \widehat{\mathcal{D}}_{\mathcal{V}}^{(m)}(D)\right)=\left\{\sum_{l \in \mathbf{N}, \underline{k} \in \mathbf{N}^{n}} \frac{a_{l, \underline{k}}}{f^{l}} \underline{\underline{k}}^{\langle\underline{k}\rangle_{(m)}}, a_{l, \underline{k}} \in \Gamma\left(\mathcal{V}, \mathcal{O}_{\mathcal{V}}\right) \mid v_{p}\left(a_{l, \underline{k}}\right) \geq \nu_{m}(l)\right. \\
\text { and } \left.v_{p}\left(a_{l, \underline{k}}\right)-\nu_{m}(l) \rightarrow+\infty \text { if }|\underline{k}|+l \rightarrow+\infty\right\} .
\end{array}
$$

Then there are canonical injective morphisms $\widehat{\mathcal{D}}_{\mathcal{V}, \mathbf{Q}}^{(m)}(D) \subset \widehat{\mathcal{D}}_{\mathcal{V}, \mathbf{Q}}^{(m+1)}(D)$ and

$$
\mathcal{D}_{\mathcal{V}}^{\dagger}\left({ }^{\dagger} D\right)=\underset{m}{\lim } \widehat{\mathcal{D}}_{\mathcal{V}, \mathbf{Q}}^{(m)}(D) .
$$

If $D=\emptyset$ the previous sheaves are simply denoted $\mathcal{D}_{\mathcal{V}}^{(m)}$ and $\widehat{\mathcal{D}}_{\mathcal{V}}^{(m)}$.

\subsection{Inequalities}

We finally recall the following inequalities for $|\underline{k}|$ and $|\underline{l}|$ elements of $\mathbf{N}^{n}, l$ and $r$ in $\mathbf{N}$.

$$
\begin{aligned}
\frac{|\underline{k}|}{p-1}-n \log _{p}(|\underline{k}|+1)-n & \leq v_{p}(\underline{k} !) \leq \frac{|\underline{k}|}{p-1} \\
\frac{|\underline{k}|}{p^{m}(p-1)}-n \log _{p}(|\underline{k}|+1)-n \frac{p}{p-1} & \leq v_{p}\left(q_{\underline{k}}^{(m)} !\right) \leq \frac{|\underline{k}|}{p^{m}(p-1)} \\
\frac{|\underline{l}|}{p^{m+1}} & \leq \nu_{m}(\underline{l}) \leq \frac{|\underline{l}|}{p^{m+1}}+n \\
0 & \leq \nu_{m}(\underline{l})-\nu_{m}(|\underline{l}|) \leq n \\
0 & \leq v_{p}\left(\left(\begin{array}{l}
l \\
r
\end{array}\right)\right) \leq p\left(\log _{p}(l)+1\right) .
\end{aligned}
$$

\subsection{An equivalence of categories}

By definition an induced $\mathcal{D}_{\mathcal{V}}^{\dagger}(D)$-module is a $\mathcal{D}_{\mathcal{V}}^{\dagger}(D)$ of the type

$$
\mathcal{D}_{\mathcal{V}}^{\dagger}(D) \otimes_{\mathcal{O}_{\mathcal{V}}(\dagger D)} \mathcal{E}
$$

where $\mathcal{E}$ is a coherent $\mathcal{O}_{\mathcal{V}}\left({ }^{\dagger} D\right)$-module and where the $\mathcal{D}_{\mathcal{V}}^{\dagger}(D)$-module structure comes from the one of $\mathcal{D}_{v}^{\dagger}(D)$. 
Coming back to the situation of the introduction, let $p_{1}$ and $p_{2}$ be the two projections $z \rightarrow y$ and $z \rightarrow y^{\vee}$, and $\infty^{\prime}=p_{2}^{-1} \infty_{y v}$

Let us now recall how to describe the structure of the category of left $\mathcal{D}_{y}^{\dagger}\left(\infty_{y}\right)$-modules, resp. $\mathcal{D}_{z}^{\dagger}(\infty)$-coherent modules, resp. $\mathcal{D}_{z}^{\dagger}\left(\infty^{\prime}\right)$-coherent modules.

Let us first start with coherent $\mathcal{D}_{y}^{\dagger}(\infty y)$-modules (see Huy95b). First note that

$$
\Gamma\left(y, \mathcal{O}_{y}\left({ }^{\dagger} \infty \mathrm{y}\right)\right)=\left\{\sum_{l} b_{l} x^{l}, b_{l} \in K, \text { and } \exists C>0, \eta<\left.1|| b_{l}\right|_{p}<C \eta^{l}\right\}
$$

and set

$$
A_{1}(K)^{\dagger}=\left\{\sum_{k \in \mathbf{N}, l \in \mathbf{N}} a_{l, k} x^{l} \partial_{x}^{[k]}, a_{l, k} \in K, \text { and } \exists C>0, \eta<\left.1|| a_{\underline{k}, \underline{l}}\right|_{p}<C \eta^{l+k}\right\},
$$

the weak completion of the Weyl algebra. It is a coherent algebra and we have ( Huy95b])

Theorem 1.5.1. The functor $\Gamma(y,$.$) (resp. R \Gamma(y,)$.$) establishes an equivalence of cate-$ gories between the category of left coherent $\mathcal{D}_{y}^{\dagger}(\infty y)$-modules (resp. $D_{\text {coh }}^{b}\left(\mathcal{D}_{y}^{\dagger}(\infty y)\right)$ ) and the category of left coherent $A_{1}(K)^{\dagger}$-modules (resp. $D_{\text {coh }}^{b}\left(A_{1}(K)^{\dagger}\right)$.

In particular, $A_{1}(K)^{\dagger} \simeq \Gamma\left(y, \mathcal{D}_{y}^{\dagger}\left(\infty_{y}\right)\right)$ and every coherent $\mathcal{D}_{y}^{\dagger}\left(\infty_{y}\right)$ admits globally over $y$ a resolution by globally projective and finitely generated $\mathcal{D}_{y}^{\dagger}(\infty y)$-modules. This resolution can be taken finite since $A_{1}(K)^{\dagger}$ has finite cohomological dimension ([NH07]).

Consider now the situation over $z$ for $m$ fixed. In theorem 3 of [Huy95b, we proved that the elements $\partial_{x}^{\left\langle k_{1}\right\rangle_{(m)}}$ are global sections over $\mathbb{P}_{\mathcal{S}}^{1}$ of the sheaves $\mathcal{D}_{\mathbb{P}_{\mathcal{S}}^{1}}^{(m)}$, so that

$$
\partial_{x}^{\left\langle k_{1}\right\rangle_{(m)}} \partial_{y}^{\left\langle k_{2}\right\rangle_{(m)}} \in \Gamma\left(z, \mathcal{D}_{z}^{(m)}\right)
$$

Moreover, an easy computation (2.1 of [Huy95b]) shows that elements $p^{\nu_{m}\left(l_{1}\right)} x^{l_{1}}$ for $l_{1} \geq 0$ are global sections of $\widehat{\mathcal{B}}_{\mathbb{P}_{\mathcal{S}}^{1}}^{(m)}$, implying that

$$
p^{\nu_{m}(\underline{l})} x^{l_{1}} y^{l_{2}} \in \Gamma\left(z, \widehat{\mathcal{B}}_{z}^{(m)}\right)
$$

Define

$$
\begin{aligned}
& \widehat{E}^{(m)}=\left\{\sum_{\underline{k} \in \mathbf{N}^{2}, \underline{l} \in \mathbf{N}^{2}} a_{\underline{l}, \underline{\underline{k}}} x^{l_{1}} y^{l_{2}} \partial_{x}^{\left\langle k_{1}\right\rangle} \partial_{y}^{\left\langle k_{2}\right\rangle}, a_{\underline{l}, \underline{k}} \in V, \text { and } v_{p}\left(a_{\underline{l}, \underline{k}}\right) \geq \nu_{m}(\underline{l})\right. \\
& \left.\mid v_{p}\left(a_{\underline{l}, \underline{k}}\right)-\nu_{m}(\underline{l}) \rightarrow+\infty \text { if }|\underline{l}|+|\underline{k}| \rightarrow+\infty\right\} .
\end{aligned}
$$


From these observations and the fact that $\widehat{\mathcal{D}}_{\mathcal{z}}^{(m)}$ is a sheaf of $p$-adically complete algebras, we see that

$$
\widehat{E}^{(m)} \subset \Gamma\left(\mathcal{Z}, \widehat{\mathcal{D}}_{\mathcal{z}}^{(m)}(\infty)\right)
$$

Consider the weak completion of the Weyl algebra in 2 variables

$$
A_{2}(K)^{\dagger}=\left\{\sum_{\underline{k} \in \mathbf{N}^{2}, \underline{l} \in \mathbf{N}^{2}} a_{\underline{l}, \underline{k}} x^{l_{1}} y^{l_{2}} \partial_{x}^{\left[k_{1}\right]} \partial_{y}^{\left[k_{2}\right]}, a_{l, k} \in K, \text { and } \exists C>0, \eta<1|| a_{\underline{k}, \underline{l}} \mid<C \eta^{|\underline{l}|+|\underline{k}|}\right\} \text {, }
$$

which is coherent from Huy95b. It is easy to see that

$$
A_{2}(K)^{\dagger}=\underset{m}{\lim } \widehat{E}_{\mathbf{Q}}^{(m)} .
$$

We endow $A_{2}(K)^{\dagger}$ with the inductive limit topology coming from this filtration.

Because the divisor $\infty$ over $z$ is ample, we can apply 4.5.1 of Huy03 which tells us that

$$
\Gamma\left(Z, \mathcal{D}_{Z}^{\dagger}(\infty)\right) \simeq A_{2}(K)^{\dagger}
$$

Moreover we have the following theorem (4.5.1 of Huy03 and 5.3.4 of Huy98).

Theorem 1.5.2. The functor $\Gamma(z,$.$) (resp. R \Gamma(\mathcal{Z},)$.$) establishes an equivalence of cate-$ gories between the category of left coherent $\mathcal{D}_{Z}^{\dagger}(\infty)$-modules (resp. $D_{\text {coh }}^{b}\left(\mathcal{D}_{\mathcal{Z}}^{\dagger}(\infty)\right)$ ) and the category of left coherent $A_{2}(K)^{\dagger}$-modules (resp. $D_{\text {coh }}^{b}\left(A_{2}(K)^{\dagger}\right)$ ).

Consider now the scheme $z=y \times y \vee$ endowed with the divisor $\infty^{\prime}=p_{2}^{-1}(\infty y \vee)$ and $\mathcal{D}_{z}^{\dagger}\left(\infty^{\prime}\right)$ the ring of arithmetic differential operators with overconvergent coefficients along $\infty^{\prime}$. In order to deal with coherent $\mathcal{D}_{\mathcal{Z}}^{\dagger}\left(\infty^{\prime}\right)$-modules, denote

$B_{2}(K)^{\dagger}=\left\{\sum_{l_{2} \in \mathbf{N}, \underline{k} \in \mathbf{N}^{2}} a_{l_{2}, \underline{k}} y^{l_{2}} \partial_{x}^{\left[k_{1}\right]} \partial_{y}^{\left[k_{2}\right]}, a_{l_{2}, \underline{k}} \in K\right.$, and $\left.\exists C>0, \eta<\left.1|| a_{l_{2}, \underline{k}}\right|_{p}<C \eta^{l_{2}+|\underline{k}|}\right\}$,

which we endow with the induced topology of $A_{2}(K)^{\dagger}$. Consider $\widehat{F}^{(m)}=\widehat{E}^{(m)} \cap B_{2}(K)^{\dagger}$. As before we observe that

$$
\widehat{F}^{(m)} \subset \Gamma\left(z, \widehat{\mathcal{D}}_{z}^{(m)}\left(\infty^{\prime}\right)\right)
$$

which leads to the following inclusion

$$
\underset{m}{\lim _{\mathrm{Q}}} \widehat{F}_{\mathbf{Q}}^{(m)}=B_{2}(K)^{\dagger} \subset \Gamma\left(\mathcal{Z}, \mathcal{D}_{\mathcal{Z}}^{\dagger}\left(\infty^{\prime}\right)\right)
$$

We apply 2.3.3 of [NH04] to see that this is actually an equality.

Finally, we will also use the following division lemma (4.3.4.2 of [NH04]). 
Theorem 1.6. $\quad i$ For any $P \in A_{2}(K)^{\dagger}$ there exists a unique $(Q, R) \in A_{2}(K)^{\dagger} \times B_{2}(K)^{\dagger}$ such that $P=Q\left(-\partial_{y}+\pi x\right)+R$.

ii The maps $P \mapsto Q$ and $P \mapsto R$ are continuous. More precisely, if $P \in \widehat{E}_{\mathbf{Q}}^{(m)}$, then $Q \in \widehat{E}_{\mathbf{Q}}^{(m+2)}$, and $R \in \widehat{E}_{\mathbf{Q}}^{(m+2)} \cap B_{2}(K)^{\dagger}$.

\section{Fourier transforms}

\subsection{Kernel of the Fourier transform}

In dimension 1 , the duality pairing $x \times X^{\vee} \rightarrow \widehat{\mathbb{A}}_{\mathcal{S}}^{1}$ is given by $t \mapsto x y$ where $t$ is the global coordinate on the right-hand side. It extends to $\delta: z=y \times y \vee \rightarrow \widehat{\mathbb{P}}_{\mathcal{S}}^{1}$, by the formula $t^{-1} \mapsto x^{-1} y^{-1}$ on neighborhoods of $\infty$. Let $L_{\pi}$ be the realization over $\widehat{\mathbb{P}}_{\mathcal{S}}^{1}$ of the overconvergent Dwork $F$-isocristal. It is given by a connection $\nabla(1)=-\pi d t$. We define $K_{\pi}$ to be the $\mathcal{D}_{z, \mathbf{Q}}^{\dagger}(\infty)$-module associated to the overconvergent $F$-isocristal $\delta^{*}\left(L_{\pi}\right)$. The module $K_{\pi}$ is thus isomorphic to $\mathcal{O}_{z}\left({ }^{\dagger} \infty\right)$ with a connection on $y \times y^{\vee}$ defined by

$$
\nabla(1)=-\pi(x d y+y d x)
$$

\subsection{Explicit descriptions of cohomological operations}

Let us consider the following diagram

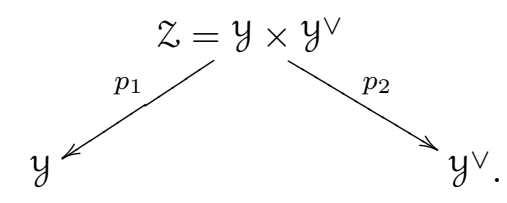

For $M, N$ two $\mathcal{O}_{\mathcal{Z}}\left({ }^{\dagger} \infty\right)$-modules, denote

$$
M \widetilde{\otimes} N=M \otimes_{\mathcal{O}_{z}\left({ }^{\dagger} \infty\right)}^{\mathbf{L}} N[-2] .
$$

Note that the sheaves $p_{1}^{-1} \mathcal{D}_{y}^{\dagger}(\infty y)$ (resp. $\left.p_{2}^{-1} \mathcal{D}_{y v}^{\dagger}(\infty y v)\right)$, are canonicaly subsheaves of rings of $\mathcal{D}_{z}^{\dagger}(\infty)$. Cohomological operations involve sheaves $\mathcal{D}_{z \rightarrow y}^{\dagger}(\infty)$, respectively $\mathcal{D}_{y_{\leftarrow}}^{\dagger}(\infty)$, which are left (resp. right) coherent $\mathcal{D}_{z}^{\dagger}(\infty)$-modules and right (resp. left) $p_{1}^{-1} \mathcal{D}_{y}^{\dagger}\left(\infty_{y}\right)$-modules, which can be explicitely described in our case. The module structures over $\mathcal{D}_{y_{\leftarrow}}^{\dagger}(\infty)$ are obtained from these of $\mathcal{D}_{z \rightarrow y}^{\dagger}(\infty)$ by twisting by the adjoint operator (1.3 of [Ber00]). In particuler underlying abelian groups of both sheaves are the same. Because the sheaves

$$
\mathcal{O}_{z}\left({ }^{\dagger} \infty\right) \otimes_{\mathcal{O}_{z}} \omega_{z} \text { and } \mathcal{O}_{z}\left({ }^{\dagger} \infty\right) \otimes_{p_{1}^{-1} \mathcal{O}_{y}} p_{1}^{-1} \omega_{y}
$$


are free, the twisted actions are easy to describe globally. For example, for $P \in \mathcal{D}_{y_{\leftarrow}}^{\dagger}(\infty)$, the right action of $\partial_{x}$ over $P$ is equal to the left action by $-\partial_{x}$ over $P$ seen as an element of $\mathcal{D}_{z \rightarrow y}^{\dagger}(\infty)$.

From 4.2.1 of [NH04], we know that these sheaves admit a free resolution, as $\mathcal{D}_{\mathcal{Z}}^{\dagger}(\infty)$ modules

$$
\begin{gathered}
0 \longrightarrow \mathcal{D}_{Z}^{\dagger}(\infty) \longrightarrow \mathcal{D}_{Z}^{\dagger}(\infty) \longrightarrow 0 \\
P \longmapsto P \partial_{y} .
\end{gathered}
$$

For the sheaf $\mathcal{D}_{\mathcal{L} \leftarrow \mathcal{z}}^{\dagger}(\infty)$, consider the map $P \mapsto \partial_{y} P$. Actually, if we endow $\mathcal{D}_{\mathcal{Z}}^{\dagger}(\infty)$ with the canonical structure of right (resp. left)- $p^{-1} \mathcal{D}_{y}^{\dagger}(\infty y)$-module this complex is a resolution of $\mathcal{D}_{z \rightarrow y}^{\dagger}(\infty)$ (resp. $\left.\mathcal{D}_{y \leftarrow z}^{\dagger}(\infty)\right)$ which is $\mathcal{D}_{z}^{\dagger}(\infty) \times \mathcal{D}_{y}^{\dagger}(\infty y)$ (resp. $\left.\mathcal{D}_{y}^{\dagger}(\infty y)\right) \times \mathcal{D}_{z}^{\dagger}(\infty)$-linear).

Moreover, thanks to 1.5 .2 the sheaves $\mathcal{D}_{z \rightarrow y}^{\dagger}(\infty)$ and $\mathcal{D}_{y_{\leftarrow}}^{\dagger}(\infty)$ are acyclic for the global section functor.

Let us also remark that both sheaves $\mathcal{D}_{y_{\leftarrow} z}^{\dagger}(\infty)$ and $\mathcal{D}_{z \rightarrow y}^{\dagger}(\infty)$ can be considered as subsheaves of $\mathcal{D}_{z}^{\dagger}(\infty)$. For example, $\mathcal{D}_{z \rightarrow y}^{\dagger}(\infty)$ is a $\mathcal{D}_{z}^{\dagger}(\infty)$-coherent module, and its global sections over $z$ are the sections of $A_{2}(K)^{\dagger}$ with no term in $\partial_{x}$.

This is the same for $\mathcal{D}_{y_{\leftarrow}}^{\dagger}(\infty)$ once we have twisted the two actions of $\mathcal{D}_{\mathcal{Z}}^{\dagger}(\infty)$ over itself by the adjoint operator.

For $M \in D_{c o h}^{b}\left(\mathcal{D}_{y}^{\dagger}(\infty y)\right)$ we state as usual

$$
p_{1}^{!}(M)=\mathcal{D}_{z \rightarrow y}^{\dagger}(\infty) \otimes_{p_{1}^{-1} \mathcal{D}_{y}^{\dagger}(\infty y)}^{\mathbf{L}} p_{1}^{-1} M[1] \in D_{c o h}^{b}\left(\mathcal{D}_{z}^{\dagger}(\infty)\right)
$$

and for $N \in D_{c o h}^{b}\left(\mathcal{D}_{\mathcal{Z}}^{\dagger}(\infty)\right)$

$$
p_{2+}(N)=\mathbf{R} p_{2 *}\left(\mathcal{D}_{y^{\vee} \leftarrow z}^{\dagger}(\infty) \otimes_{\mathcal{D}_{z}^{\dagger}(\infty)}^{\mathbf{L}} N\right)
$$

whose cohomology sheaves are not coherent in general. Fourier transform can now be defined.

\subsection{Fourier transform}

Definition 2.3.1. For $M \in D_{c o h}^{b}\left(\mathcal{D}_{y}^{\dagger}\left(\infty_{y}\right)\right)$

$$
\mathcal{F}(M)=p_{2+}\left(p_{1}^{!} M \widetilde{\otimes} K_{\pi}\right) .
$$

Let us recall the fundamental results of [NH04]. 
Theorem 2.3.2. $\quad i$ (4.3.4 of [NH04]) there is a canonical isomorphism

$$
\left.\mathcal{D}_{y \vee}^{\dagger}\left(\infty_{y \vee}\right)\right)[-1] \rightarrow \mathcal{F}\left(\mathcal{D}_{y}^{\dagger}(\infty y)\right)
$$

ii (5.3.1 of loc. cit.) If $M \in D_{\text {coh }}^{b}\left(\mathcal{D}_{y}^{\dagger}\left(\infty_{y}\right)\right)$, then $\mathcal{F}(M) \in D_{\text {coh }}^{b}\left(\mathcal{D}_{y v}^{\dagger}\left(\infty_{y v}\right)\right)$.

\subsection{Fourier transform with compact support}

To define Fourier transform with compact support, we will need to work with $\mathcal{D}_{\mathcal{Z}}^{\dagger}\left(\infty^{\prime}\right)$ modules. In particular, we will use the cohomological functor $p_{2+}^{\prime}: D_{\text {coh }}^{b}\left(\mathcal{D}_{\mathcal{Z}}^{\dagger}\left(\infty^{\prime}\right)\right) \rightarrow$ $D_{c o h}^{b}\left(\mathcal{D}_{\mathrm{yv}}^{\dagger}\left(\infty_{\mathrm{yv}}\right)\right)$, which preserves coherence, since $p_{2}$ is proper and thanks to the fact that $\infty^{\prime}=p_{2}^{-1}(\infty)$.

We will also use the scalar restriction functor $\rho: D^{b}\left(\mathcal{D}_{\mathcal{Z}}^{\dagger}(\infty)\right) \rightarrow D^{b}\left(\mathcal{D}_{z}^{\dagger}\left(\infty^{\prime}\right)\right)$. For $M$ in $D_{c o h}^{b}\left(\mathcal{D}_{\mathcal{Z}}^{\dagger}(\infty)\right)$, we denote the dual

$$
\mathbf{D}_{\mathcal{Z}}(M)=\mathbf{R} \mathcal{H} \operatorname{Lom}_{\mathcal{D}_{\mathcal{Z}}^{\dagger}(\infty)}\left(M, \mathcal{D}_{\mathcal{Z}}^{\dagger}(\infty) \otimes_{\mathcal{O}_{\mathcal{Z}}} \omega_{\mathcal{Z}}^{-1}[2]\right) \in D_{c o h}^{b}\left(\mathcal{D}_{\mathcal{Z}}^{\dagger}(\infty)\right)
$$

since the sheaf $\mathcal{D}_{\mathcal{Z}}^{\dagger}(\infty)$ has finite cohomological dimension ([NH07]), and the corresponding dual functor $\mathbf{D}_{z}^{\prime}(M)$, for $M \in D_{c o h}^{b}\left(\mathcal{D}_{\mathcal{z}}^{\dagger}\left(\infty^{\prime}\right)\right.$ ), (resp. $\mathbf{D}_{y}$ and $\mathbf{D}_{y \vee}$ for $\mathcal{D}_{y}^{\dagger}(\infty y)$, resp. $\mathcal{D}_{y v}^{\dagger}(\infty y v)$-modules).

The following division lemma will be crucial.

Lemma 2.4.1. Let $\mathfrak{U}=D_{+}\left(u_{1}\right) \times D_{+}\left(v_{0}\right)$ or $\mathcal{U}=D_{+}\left(u_{1}\right) \times D_{+}\left(v_{1}\right)$ and $x^{\prime}=1 / x$.

$i$ The elements $\left(-x^{\prime} \partial_{y}+\pi\right)$ and $-\partial_{y}+\pi x$ generate the same left ideal of $\mathcal{D}_{\mathcal{Z}}^{\dagger}(\infty)(\mathcal{U})$.

ii For any $P \in \mathcal{D}_{\mathcal{Z}}^{\dagger}(\infty)(\mathcal{U})$ there exists $(Q, R) \in \mathcal{D}_{\mathcal{Z}}^{\dagger}(\infty)(\mathcal{U}) \times \mathcal{D}_{\mathcal{Z}}^{\dagger}\left(\infty^{\prime}\right)(\mathcal{U})$ such that $P=Q\left(-x^{\prime} \partial_{y}+\pi\right)+R$.

iii The previous decomposition is not unique. But, if $Q\left(-\partial_{y}+\pi x\right) \in \mathcal{D}_{\mathcal{Z}}^{\dagger}\left(\infty^{\prime}\right)(\mathcal{U})$, then $Q \in \mathcal{D}_{z}^{\dagger}\left(\infty^{\prime}\right)(\mathcal{U})$.

iv If $Q \in \mathcal{D}_{\mathcal{Z}}^{\dagger}(\infty)(\mathcal{U})$ and $Q\left(-\partial_{y}+\pi x\right)=0$, then $Q=0$.

Remark. Analogous statements hold for the left multiplication by $-\partial_{y}+\pi x$, or $\partial_{y}+\pi x$, considering right ideals generated by these elements.

Proof. Recall that $x \in \Gamma\left(\mathcal{Z}, \mathcal{O}_{z}\left({ }^{\dagger} \infty\right)\right)$. The first assertion comes from the equality

$$
x\left(-x^{\prime} \partial_{y}+\pi\right)=-\partial_{y}+\pi x .
$$


The open set $D_{+}\left(u_{1}\right) \times D_{+}\left(v_{0}\right)$ will be endowed with coordinates $x^{\prime}=1 / x$ and $t=y$, and $D_{+}\left(u_{1}\right) \times D_{+}\left(v_{1}\right)$ with coordinates $x^{\prime}$ and $t=1 / y$. Denote $D^{\dagger}=\mathcal{D}_{\mathcal{Z}}^{\dagger}(\infty)(\mathcal{U})$, and $D^{\prime \dagger}=\mathcal{D}_{\mathcal{Z}}^{\dagger}\left(\infty^{\prime}\right)(\mathcal{U})$, which contains the algebras $\widehat{F}_{\mathrm{Q}}^{(m)}$ described in 1.5 .

By considering the right $\mathcal{O}_{z}$-module structure on $\mathcal{D}_{z}^{\dagger}(\infty)$, we see that an element $Q \in D^{\dagger}$ can be written

$$
Q=\sum_{\underline{k} \in \mathbf{N}^{2}, \underline{l} \in \mathbf{Z}^{2}} a_{\underline{l}, \underline{k}} \partial_{x^{\prime}}^{\left[k_{1}\right]} \partial_{t}^{\left[k_{2}\right]} x^{\prime l_{1}} t^{l_{2}}
$$

such that

$$
a_{\underline{l}, \underline{k}} \in K \text { and } \exists C>0, M>0, \mid v_{p}\left(a_{\underline{l}, \underline{k}}\right) \geq \frac{\max \left\{-l_{1}, 0\right\}+\max \left\{-l_{2}, 0\right\}+|\underline{k}|}{C}-M,
$$

(note that on $D_{+}\left(u_{1}\right) \times D_{+}\left(v_{0}\right)$ coefficients $a_{\left(l_{1}, l_{2}\right), \underline{k}}$ are equal to 0 if $\left.l_{2}<0\right)$ ). With this notation $Q \in D^{\prime \dagger}$ if and only if $\forall \underline{k} \in \mathbf{N}^{2}, \forall \underline{l} \mid l_{1}<0, a_{\underline{l}, \underline{k}}=0$. We can also write $Q$ this way

$$
Q=\sum_{l_{1} \in \mathbf{Z}} Q_{l_{1}} x^{l_{1}}
$$

with

$$
Q_{l_{1}}=\sum_{\substack{k=\left(k_{1}, k_{2}\right) \in \mathbf{N} \times \mathbf{N} \\ l_{2} \in \mathbf{Z}}} a_{\left(l_{1}, l_{2}\right), \underline{k}} \partial_{x^{\prime}}^{\left[k_{1}\right]} \partial_{t}^{\left[k_{2}\right]} t^{l_{2}} \in \Gamma\left(\mathcal{U}, \mathcal{D}_{\mathcal{Z}}^{\dagger}\left(\infty^{\prime}\right)\right) .
$$

Let us observe that the condition (10) is equivalent to the fact that there exist $m>0$ such that

$$
Q_{l_{1}} \in \Gamma\left(\mathcal{U}, \widehat{\mathcal{D}}_{\mathcal{Z}, \mathbf{Q}}^{(m)}\left(\infty^{\prime}\right)\right)
$$

and elements $T_{l_{1}}$ of $\Gamma\left(\mathcal{U}, \widehat{\mathcal{D}}_{z}^{(m)}\left(\infty^{\prime}\right)\right)$ such that

$$
Q_{l_{1}}=u_{l_{1}} p^{\nu_{m}\left(-l_{1}\right)} T_{l_{1}} \text {, for some } u_{l_{1}} \in K \text { satisfying } v_{p}\left(u_{l_{1}}\right) \rightarrow+\infty \text { if }\left|l_{1}\right| \rightarrow+\infty \text {. }
$$

We define

$$
R^{\prime \prime}=\sum_{l_{1} \in \mathbf{N}} Q_{l_{1}} x^{\prime l_{1}} \in \Gamma\left(\mathcal{U}, \widehat{\mathcal{D}}_{\mathcal{Z}, \mathbf{Q}}^{(m)}\left(\infty^{\prime}\right)\right),
$$

so that we can write down

$$
Q=\sum_{l_{1}<0} u_{l_{1}} p^{\nu_{m}\left(\left|l_{1}\right|\right)} T_{l_{1}} x^{\left|l_{1}\right|}+R^{\prime \prime}
$$

with $T_{l_{1}} \in \Gamma\left(\mathcal{U}, \widehat{\mathcal{D}}_{\mathcal{Z}, \mathbf{Q}}^{(m)}(\infty)\right)$ and $v_{p}\left(u_{l_{1}}\right) \rightarrow+\infty$ if $\left|l_{1}\right| \rightarrow+\infty$.

We will first prove (iii). Suppose that $Q\left(-\partial_{y}+\pi x\right) \in \Gamma\left(\mathcal{U}, \mathcal{D}_{\mathcal{Z}}^{\dagger}\left(\infty^{\prime}\right)\right)$. Because of (3)), we can suppose that

$$
Q=\sum_{l_{1}<0} Q_{l_{1}} x^{l_{1}}
$$


Now we are reduced to prove that $Q=0$, that we can do in restriction to $D_{+}\left(u_{1}\right) \times D_{+}\left(v_{0} v_{1}\right)$, on which we choose $x^{\prime}$ and $y$ as coordinates.

Let us compute

$$
Q\left(-\partial_{y}+\pi x\right)=\sum_{l_{1}<0}\left(-Q_{l_{1}} \partial_{y}+\pi Q_{l_{1}+1}\right) x^{l_{1}}
$$

Therefore, $Q\left(-\partial_{y}+\pi x\right)$ is an element of $\Gamma\left(\mathcal{U}, \mathcal{D}_{\mathcal{Z}}^{\dagger}\left(\infty^{\prime}\right)\right)$ if and only if

$$
\begin{gathered}
Q_{-1} \partial_{y}=0, \text { and } \\
\forall l_{1} \leq-1,-Q_{l_{1}} \partial_{y}+\pi Q_{l_{1}+1}=0 .
\end{gathered}
$$

Let us decompose

$$
Q_{-1}=\sum_{\underline{k}} \beta_{\underline{k}}(y) \partial_{x^{\prime}}^{\left[k_{1}\right]} \partial_{y}^{\left[k_{2}\right]},
$$

with $\beta_{\underline{k}}(y) \in \mathcal{O}_{\mathcal{Z}, \mathbf{Q}}\left(D_{+}\left(v_{0} u_{0} u_{1}\right)\right)$, then we compute

$$
Q_{-1} \partial_{y}=\sum_{\underline{k} \mid k_{2} \geq 1} k_{2} \beta_{\left(k_{1}, k_{2}-1\right)}(y) \partial_{x^{\prime}}^{\left[k_{1}\right]} \partial_{y}^{\left[k_{2}\right]},
$$

which is nul if and only if $\forall \underline{k}, \beta_{\underline{k}}=0$, i.e. $Q_{-1}=0$. Thus by descending induction, one sees from (5) that $\forall l_{1} \leq-1, Q_{l_{1}}=0$, and $Q=0$.

Let us prove now (ii), the existence of the decomposition. Recall that $\partial_{y} \in \Gamma\left(z, \mathcal{D}_{z}^{\dagger}\left(\infty^{\prime}\right)\right)$ and $\left(-\partial_{y}+\pi x\right) \in \Gamma\left(\mathcal{Z}, \mathcal{D}_{\mathcal{Z}}^{\dagger}(\infty)\right)$. Since these two elements commute we have the following equalities

$$
\begin{aligned}
p^{\nu_{m}\left(\left|l_{1}\right|\right)} x^{\left|l_{1}\right|}= & \frac{p^{\nu_{m}\left(\left|l_{1}\right|\right)}}{\pi^{\left|l_{1}\right|}}\left(\left(-\partial_{y}+\pi x\right)+\partial_{y}\right)^{\left|l_{1}\right|} \\
= & \frac{p^{\nu_{m}\left(\left|l_{1}\right|\right)}}{\pi^{\left|l_{1}\right|}\left|l_{1}\right| ! \partial_{y}^{\left[\left|l_{1}\right|\right]}} \\
& +\left[\sum_{r=1}^{\left|l_{1}\right|} \sum_{s=0}^{r-1}(-1)^{r-1+s} \frac{p^{\nu_{m}\left(\left|l_{1}\right|\right)}}{\pi^{\left|l_{1}\right|-s}}\left(\begin{array}{c}
\left|l_{1}\right| \\
r
\end{array}\right)\left(\begin{array}{c}
r-1 \\
s
\end{array}\right)\left(\left|l_{1}\right|-1-s\right) ! \partial_{y}^{\left[\left|l_{1}\right|-1-s\right]} x^{s}\right]\left(-\partial_{y}+\pi x\right) \\
= & \frac{p^{\nu_{m}\left(\left|l_{1}\right|\right)}}{\pi^{\left|l_{1}\right|}} \frac{\left|l_{1}\right| !}{q_{\left|l_{1}\right|}^{(m+2)} !} \partial_{y}^{\left\langle\left|l_{1}\right|\right\rangle_{(m+2)}} \\
& +\left[\sum_{r=1}^{\left|l_{1}\right|} \sum_{s=0}^{r-1}(-1)^{r-1+s} \frac{p^{\nu_{m}\left(\left|l_{1}\right|\right)}}{\pi^{\left|l_{1}\right|-s}}\left(\begin{array}{c}
\left|l_{1}\right| \\
r
\end{array}\right)\left(\begin{array}{c}
r-1 \\
s
\end{array}\right) \frac{\left(\left|l_{1}\right|-1-s\right) !}{q_{\left|l_{1}\right|-1-s}^{(m+2)} !} \partial_{y}^{\left\langle\left|l_{1}\right|-1-s\right\rangle_{(m+2)} s} x^{s}\right]\left(-\partial_{y}+\right.
\end{aligned}
$$

Denote

$$
R_{l_{1}}=\frac{p^{\nu_{m}\left(\left|l_{1}\right|\right)}}{\pi^{\left|l_{1}\right|}} \frac{\left|l_{1}\right| !}{q_{\left|l_{1}\right|}^{(m+2)} !} \partial_{y}^{\left\langle\left|l_{1}\right|\right\rangle_{(m+2)}}
$$




$$
\begin{gathered}
S_{l_{1}}=\sum_{r=1}^{\left|l_{1}\right|} \sum_{s=0}^{r-1}(-1)^{r-1+s} \frac{p^{\nu_{m}\left(\left|l_{1}\right|\right)}}{\pi^{\left|l_{1}\right|-s}}\left(\begin{array}{c}
\left|l_{1}\right| \\
r
\end{array}\right)\left(\begin{array}{c}
r-1 \\
s
\end{array}\right) \frac{\left(\left|l_{1}\right|-1-s\right) !}{q_{\left|l_{1}\right|-1-s}^{(m+2)} !} \partial_{y}^{\left\langle\left|l_{1}\right|-1-s\right\rangle_{(m+2)}} x^{s}, \\
c_{l_{1}}(r, s)=(-1)^{r-1+s} \frac{p^{\nu_{m}\left(\left|l_{1}\right|\right)}}{\pi^{\left|l_{1}\right|-s}}\left(\begin{array}{c}
\left|l_{1}\right| \\
r
\end{array}\right)\left(\begin{array}{c}
r-1 \\
s
\end{array}\right) \frac{\left(\left|l_{1}\right|-1-s\right) !}{q_{\left|l_{1}\right|-1-s}^{(m+2)} !} .
\end{gathered}
$$

By definition, we have the following relation

$$
p^{\nu_{m}\left(\left|l_{1}\right|\right)} x^{\left|l_{1}\right|}=S_{l_{1}}\left(-\partial_{y}+\pi x\right)+R_{l_{1}} .
$$

Then, from estimations 1.4 we see that

$$
v_{p}\left(\frac{p^{\nu_{m}\left(\left|l_{1}\right|\right)}}{\pi^{\left|l_{1}\right|}} \frac{\left|l_{1}\right| !}{q_{\left|l_{1}\right|}^{(m+2)} !}\right) \geq \frac{p^{2}-p-1}{p^{m+2}(p-1)}\left|l_{1}\right|-\log _{p}\left(\left|l_{1}\right|+1\right)-1 \rightarrow \infty \text { if }\left|l_{1}\right| \rightarrow+\infty,
$$

which proves that $R_{l_{1}} \in \widehat{E}^{(m+2)}$ for $\left|l_{1}\right|$ big enough. We also see that $\forall r \leq\left|l_{1}\right|-1, s \leq r$,

$$
\begin{aligned}
v_{p}\left(c_{l_{1}}(r, s)\right) & \geq \frac{\left|l_{1}\right|}{p^{m+1}}-\frac{\left|l_{1}\right|-s}{p-1}-\log _{p}\left(\left|l_{1}\right|+1\right)+\frac{\left|l_{1}\right|-1-s}{p-1}-1-\frac{\left|l_{1}\right|-1-s}{p^{m+2}(p-1)} \\
& \geq\left(\frac{p^{2}-p-1}{p^{m+2}(p-1)}\right)\left|l_{1}\right|-\log _{p}\left(\left|l_{1}\right|+1\right)-2 \rightarrow \infty \text { if }\left|l_{1}\right| \rightarrow+\infty,
\end{aligned}
$$

which proves that $S_{l_{1}} \in \widehat{E}^{(m+2)}$ for $\left|l_{1}\right|$ big enough. As a consequence $S_{l_{1}}$ and resp. $R_{l_{1}}$ are elements of $\Gamma\left(\mathcal{U}, \widehat{\mathcal{D}}_{\mathcal{Z}}^{(m+2)}(\infty)\right)$ for $\left|l_{1}\right|$ big enough $\left(\operatorname{resp} . \Gamma\left(\mathcal{U}, \widehat{\mathcal{D}}_{\mathcal{Z}}^{(m+2)}\left(\infty^{\prime}\right)\right)\right)$.

Let $Q \in D^{\dagger}$. We can use the description given in (3). Since $\left|u_{l_{1}}\right| \rightarrow 0$ if $\left|l_{1}\right| \rightarrow+\infty$, we observe that

$$
Q^{\prime}=\sum_{l_{1}<0} u_{l_{1}} T_{l_{1}} S_{l_{1}} \in \Gamma\left(\mathcal{U}, \widehat{\mathcal{D}}_{\mathcal{Z}, \mathbf{Q}}^{(m+2)}(\infty)\right)
$$

and

$$
R^{\prime}=\sum_{l_{1}<0} u_{l_{1}} T_{l_{1}} R_{l_{1}} \in \Gamma\left(\mathcal{U}, \widehat{\mathcal{D}}_{\mathcal{Z}, \mathbf{Q}}^{(m+2)}\left(\infty^{\prime}\right)\right) .
$$

Moreover, we have the following equalities

$$
\begin{aligned}
Q & =\sum_{l_{1}<0} u_{l_{1}} p^{\nu_{m}\left(\left|l_{1}\right|\right)} T_{l_{1}} x^{\left|l_{1}\right|}+R^{\prime \prime} \\
& =\sum_{l_{1}<0} u_{l_{1}} T_{l_{1}}\left(S_{l_{1}}\left(-\partial_{y}+\pi x\right)+R_{l_{1}}\right)+R^{\prime \prime} \\
& =Q^{\prime} x\left(-x^{\prime} \partial_{y}+\pi\right)+R^{\prime}+R^{\prime \prime}
\end{aligned}
$$

which shows (ii) of the lemma. 
Now, let $Q \in D^{\dagger}$ such that $Q\left(-\partial_{y}+\pi x\right)=0$. From (iii), we know that in fact $Q \in D^{\prime \dagger}$. As in the previous case, we restrict ourselves to $\mathcal{U}=D_{+}\left(u_{0}\right) \times D_{+}\left(v_{0} v_{1}\right)$ and we decompose

$$
Q=\sum_{l_{1} \in \mathbf{N}} Q_{l_{1}} x^{l_{1}}
$$

The recursive formula (5) still holds and we get

$$
\begin{gathered}
\pi Q_{0}=0, \text { and } \\
\forall l_{1} \geq 0, \pi Q_{l_{1}+1}=Q_{l_{1}} \partial_{y} .
\end{gathered}
$$

By induction, this proves that $Q_{l_{1}}=0$ for all $l_{1} \geq 0$ and thus that $Q=0$.

The key lemma to define the Fourier transform with compact support is the following:

Lemma 2.4.2. Let $\left.M \in D_{c o h}^{b}\left(\mathcal{D}_{y}^{\dagger}\left(\infty_{y}\right)\right)\right)$, then

$$
\rho_{*} \mathbf{D}_{\mathcal{Z}}\left(p_{1}^{!} M \widetilde{\otimes} K_{\pi}\right) \in D_{c o h}^{b}\left(\mathcal{D}_{\mathcal{Z}}^{\dagger}\left(\infty^{\prime}\right)\right) .
$$

Proof. It is enough to prove this lemma in the case of a single $\mathcal{D}_{y}^{\dagger}\left(\infty_{y}\right)$ coherent module $M$. Such a module admits a resolution by direct factors of free modules of finite rank (1.5.1). It is thus enough to prove this lemma in the case of $\mathcal{D}_{y}^{\dagger}(\infty y)$ itself.

If $\mathcal{F}$ is an $\mathcal{O}_{\mathcal{Z}}$-coherent module, we denote

$$
\widetilde{\mathcal{F}}=\mathcal{F} \otimes_{\mathcal{O}_{z}} \mathcal{O}_{z}\left({ }^{\dagger} \infty\right)
$$

Then by 2.1 .1 of [NH04] $\widetilde{\mathfrak{T}}_{z / S}\left(\right.$ resp. $\left.\widetilde{\mathfrak{T}}_{z / y}\right)$ are free $\mathcal{O}_{z}\left({ }^{\dagger} \infty\right)$-modules of basis $\partial_{x}, \partial_{y}$ (resp. $\left.\partial_{y}\right)$.

Let us reformulate lemma 4.2.4 of [NH04]. Let $K$. the complex of induced $\mathcal{D}_{\mathcal{Z}}^{\dagger}(\infty)$ modules in degrees -1 and 0

$$
0 \rightarrow \mathcal{D}_{Z}^{\dagger}(\infty) \otimes_{\mathcal{O}_{z}^{\dagger}(\infty)} \Lambda^{1} \widetilde{\mathcal{T}}_{z / y} \stackrel{d}{\rightarrow} \mathcal{D}_{z}^{\dagger}(\infty) \rightarrow 0
$$

where

$$
d\left(P \otimes \partial_{y}\right)=P \cdot\left(\partial_{y}+\pi x\right) .
$$

Lemma 2.4.3. In the derived category $D_{c o h}^{b}\left(\mathcal{D}_{z}^{\dagger}(\infty)\right)$ this complex $K$. is equal to the complex

$$
p_{1}^{!} \mathcal{D}_{y}^{\dagger}(\infty y) \widetilde{\otimes}_{\mathcal{O}_{z}^{\dagger}(\infty)} K_{\pi}[1]
$$

which is nothing but the complex $p_{1}^{*} \mathcal{D}_{y}^{\dagger}(\infty y) \otimes K_{\pi}$ (in degree 0$)$. 
The augmentation map $\varepsilon^{\prime}: \mathcal{D}_{\mathcal{Z}}^{\dagger}(\infty) \rightarrow p_{1}^{*} \mathcal{D}_{y}^{\dagger}(\infty y) \otimes K_{\pi}$ is given by

$$
\begin{aligned}
\varepsilon^{\prime}\left(\partial_{y}\right) & =-\pi x \otimes 1 \\
\varepsilon^{\prime}\left(\partial_{x}\right) & =\left(\partial_{x}-\pi y\right) \otimes 1 .
\end{aligned}
$$

In the rest of the proof, we identify the left induced $\mathcal{D}_{z}^{\dagger}(\infty)$-module $\mathcal{D}_{z}^{\dagger}(\infty) \otimes_{\mathcal{O}_{z}^{\dagger}(\infty)} \Lambda^{1} \widetilde{\mathcal{T}}_{z / y}$ with $\mathcal{D}_{\mathcal{Z}}^{\dagger}(\infty)$. Then $\mathbf{R} \mathcal{H}_{o m} \mathcal{D}_{\mathcal{Z}(\infty)}^{\dagger}\left(K_{\bullet}, \mathcal{D}_{\mathcal{Z}}^{\dagger}(\infty)\right)[2]$ is represented by the following complex of right $\mathcal{D}_{\mathcal{Z}}^{\dagger}(\infty)$-modules, whose terms are in degrees -2 and -1

$$
0 \rightarrow \mathcal{D}_{z}^{\dagger}(\infty) \stackrel{d^{\prime}}{\rightarrow} \mathcal{D}_{z}^{\dagger}(\infty) \rightarrow 0
$$

such that $d^{\prime}(P)=\left(\partial_{y}+\pi x\right) P$. Finally, we see that $L_{\bullet}=\mathbf{D}\left(K_{\bullet}\right)$ is represented in $D_{\text {coh }}^{b}\left(\mathcal{D}_{\mathcal{Z}}^{\dagger}(\infty)\right)$ by the following complex in degrees -2 et -1

$$
0 \rightarrow \mathcal{D}_{z}^{\dagger}(\infty) \stackrel{d^{\prime \prime}}{\rightarrow} \mathcal{D}_{z}^{\dagger}(\infty) \rightarrow 0
$$

such that $d^{\prime \prime}(P)=P\left(-\partial_{y}+\pi x\right)$.

Consider now the canonical map

$$
\mathcal{D}_{z}^{\dagger}\left(\infty^{\prime}\right) \rightarrow \mathcal{D}_{z}^{\dagger}(\infty) / \mathcal{D}_{z}^{\dagger}(\infty)\left(-\partial_{y}+\pi x\right)
$$

Over $D_{+}\left(u_{0}\right)$ both sheaves $\mathcal{D}_{Z}^{\dagger}\left(\infty^{\prime}\right)$ and $\mathcal{D}_{Z}^{\dagger}(\infty)$ coïncide. Let us study the situation over $D_{+}\left(u_{1}\right)$. From the previous lemma 2.4.1, we see that this map is surjective over $D_{+}\left(u_{1}\right) \times$ $D_{+}\left(v_{0}\right)$ and $D_{+}\left(u_{1}\right) \times D_{+}\left(v_{1}\right)$ and that over these open subsets the following complex is exact (using notations of 2.4.1)

$$
0 \rightarrow \mathcal{D}_{z}^{\dagger}\left(\infty^{\prime}\right) \stackrel{d^{\prime \prime}}{\rightarrow} \mathcal{D}_{z}^{\dagger}\left(\infty^{\prime}\right) \rightarrow \rho_{*}\left(\mathcal{D}_{z}^{\dagger}(\infty) / \mathcal{D}_{z}^{\dagger}(\infty)\left(-\partial_{y}+\pi x\right)\right) \rightarrow 0
$$

where $d^{\prime \prime}(P)=P\left(-x^{\prime} \partial_{y}+\pi\right)$, showing that $\rho_{*} \mathbf{D}_{z}\left(K_{\bullet}\right) \in D_{\text {coh }}^{b}\left(\mathcal{D}_{\mathcal{Z}}^{\dagger}\left(\infty^{\prime}\right)\right)$, hence $\rho_{*} \mathbf{D}_{z}\left(p_{1}^{!} M \widetilde{\otimes} K_{\pi}\right) \in D_{c o h}^{b}\left(\mathcal{D}_{z}^{\dagger}\left(\infty^{\prime}\right)\right)$ for any $M \in D_{\text {coh }}^{b}\left(\mathcal{D}_{z}^{\dagger}(\infty)\right)$.

Finally, this leads us to the following definition.

Definition 2.4.4. For $M \in D_{\text {coh }}^{b}\left(\mathcal{D}_{y}^{\dagger}\left(\infty_{y}\right)\right)$

$$
\mathcal{F}_{!}(M)=p_{2+}^{\prime} \mathbf{D}_{z}^{\prime} \rho_{*} \mathbf{D}_{\mathcal{z}}\left(p_{1}^{!} M \widetilde{\otimes} K_{\pi}\right) \in D_{c o h}^{b}\left(\mathcal{D}_{\mathcal{Z}}^{\dagger}\left(\infty^{\prime}\right)\right)
$$

Note that from the previous lemma 2.4.2, we know that $\mathcal{F}_{!}(M) \in D_{\text {coh }}^{b}\left(\mathcal{D}_{\text {yv }}^{\dagger}\left(\infty_{y \vee}\right)\right)$. 


\section{Comparison theorem}

Proposition 3.1. Let $M \in D_{\text {coh }}^{b}\left(\mathcal{D}_{\mathcal{Z}}^{\dagger}(\infty)\right)$, there is a canonical map : $\mathcal{F}_{!}(M) \rightarrow \mathcal{F}(M)$.

Proof. Let $E$ be a coherent $\mathcal{D}_{\mathcal{Z}}^{\dagger}(\infty)$-module, such that $\rho_{*} E$ is a coherent $\mathcal{D}_{\mathcal{Z}}^{\dagger}\left(\infty^{\prime}\right)$-module. There are canonical maps $\mathbf{D}_{z}^{\prime}\left(\rho_{*} E\right) \rightarrow \mathbf{D}_{\mathcal{Z}}(E)$ and also canonical maps

$$
p_{2+}^{\prime} \mathbf{D}_{Z}^{\prime}(E) \rightarrow p_{2+} \mathbf{D}_{\mathcal{Z}}(E)
$$

Applying this to $E=\rho_{*} \mathbf{D}_{\mathcal{Z}}(\infty)\left(p_{1}^{!} M \widetilde{\otimes} K_{\pi}\right)$ gives a canonical map

$$
\mathcal{F}_{!}(M) \rightarrow p_{2+} \mathbf{D}_{Z} \mathbf{D}_{\mathcal{Z}}\left(p_{1}^{!} M \widetilde{\otimes} K_{\pi}\right)
$$

And we apply the biduality theorem 3.6 of [Vir00, to see that the RHS can be identified with $\mathcal{F}(M)$.

Theorem 3.2. Let $M \in D_{\text {coh }}^{b}\left(\mathcal{D}_{\mathcal{Z}}^{\dagger}(\infty)\right)$, there is a canonical isomorphism : $\mathcal{F}_{!}(M) \simeq \mathcal{F}(M)$.

Since $\mathcal{F}$ and $\mathcal{F}_{\text {! }}$ are both way-out functors, we are reduced to the case of a single module $M$. Thanks to 1.5.1, we can suppose that $M=\mathcal{D}_{y}^{\dagger}\left(\infty_{y}\right)$. This computation is the aim of the next subsection.

\subsection{Computation of $\mathcal{F}_{!}\left(\mathcal{D}_{y}^{\dagger}(\infty y)\right)$}

Let us consider $\mathcal{U}$ one of the open sets $D_{+}\left(u_{1}\right) \times D_{+}\left(v_{0}\right)$ or $D_{+}\left(u_{1}\right) \times D_{+}\left(v_{1}\right)$ of $\mathcal{Z}$, denote by $x^{\prime}$ and $t$ coordinates over $\mathcal{U}$. Then, over $\mathcal{U}$, we have the following resolution of the sheaf $\mathcal{D}_{y^{\vee} \leftarrow z}^{\dagger}\left(\infty^{\prime}\right)$ by right $\mathcal{D}_{z}^{\dagger}\left(\infty^{\prime}\right)$-modules

$$
\begin{gathered}
0 \longrightarrow \mathcal{D}_{\mathcal{Z}}^{\dagger}\left(\infty^{\prime}\right) \longrightarrow \mathcal{D}_{\mathcal{Z}}^{\dagger}\left(\infty^{\prime}\right) \longrightarrow \mathcal{D}_{y^{\vee} \leftarrow z}^{\dagger}\left(\infty^{\prime}\right) \longrightarrow 0 \\
P \longmapsto \partial_{x^{\prime}} P
\end{gathered}
$$

and exactly the same resolution for the sheaf $\mathcal{D}_{z}^{\dagger}(\infty)$, replacing $\mathcal{D}_{z}^{\dagger}\left(\infty^{\prime}\right)$ by $\mathcal{D}_{\mathcal{Z}}^{\dagger}(\infty)$. This proves that there is a canonical isomorphism of right $\mathcal{D}_{z}^{\dagger}(\infty)$-modules

$$
\mathcal{D}_{y \vee \leftarrow z}^{\dagger}\left(\infty^{\prime}\right) \otimes_{\mathcal{D}_{z}^{\dagger}\left(\infty^{\prime}\right)} \mathcal{D}_{z}^{\dagger}(\infty) \simeq \mathcal{D}_{y \vee \leftarrow z}^{\dagger}(\infty)
$$

In fact, this isomorphism is also right $\mathcal{D}_{y \vee}^{\dagger}\left(\infty_{y v}\right)$-linear, property that we can check over the open set $D_{+}\left(u_{0}\right) \times D_{+}\left(v_{0}\right)$ where the previous isomorphism coincides with identity. Now we have the following lemma. 
Lemma 3.3.1. Let $M$ be a coherent $\mathcal{D}_{z}^{\dagger}(\infty)$-module such that $\rho_{*} M$ is a coherent $\mathcal{D}_{z}^{\dagger}\left(\infty^{\prime}\right)$ module, then there is a canonical isomorphism of coherent $\mathcal{D}_{\mathcal{Z}}^{\dagger}(\infty)$-modules

$$
\mathcal{D}_{Z}^{\dagger}(\infty) \otimes_{\mathcal{D}_{Z}^{\dagger}\left(\infty^{\prime}\right)} \rho_{*} M \simeq M
$$

Proof. The canonical map of the statement is a morphism of coherent $\mathcal{D}_{\mathcal{Z}}^{\dagger}(\infty)$-modules, which is an isomorphism over $D_{+}\left(u_{0}\right) \times D_{+}\left(v_{0}\right)$ and thus an isomorphism (cf 4.3 .7 of [Ber96]).

With the hypothesis of the lemma, we get

$$
\begin{aligned}
p_{2+} M & =R p_{2 *}\left(\mathcal{D}_{y^{\vee} \leftarrow z}^{\dagger}(\infty) \otimes_{\mathcal{D}_{z}^{\dagger}(\infty)} M\right) \\
& \simeq R p_{2 *}\left(\mathcal{D}_{y^{\vee} \leftarrow z}^{\dagger}\left(\infty^{\prime}\right) \otimes_{\mathcal{D}_{\mathcal{Z}}^{\dagger}\left(\infty^{\prime}\right)} \mathcal{D}_{\mathcal{Z}}^{\dagger}(\infty) \otimes_{\mathcal{D}_{\mathcal{Z}}^{\dagger}(\infty)} M\right) \\
& \simeq R p_{2 *}\left(\mathcal{D}_{y^{\vee} \leftarrow z}^{\dagger}\left(\infty^{\prime}\right) \otimes_{\mathcal{D}_{\mathcal{Z}}^{\dagger}\left(\infty^{\prime}\right)} \rho_{*}(M)\right) \\
& \simeq p_{2+}^{\prime} M .
\end{aligned}
$$

Applying this to $M=K_{\bullet}[-1]$ of 2.4 .3 , we see that

$$
\begin{aligned}
\mathcal{F}_{!}\left(\mathcal{D}_{y}^{\dagger}\left(\infty_{y}\right)\right) & =p_{2+}^{\prime} \mathbf{D}_{z}^{\prime} \rho_{*} \mathbf{D}_{z}\left(K_{\bullet}[-1]\right) \\
& \simeq \mathbf{D}_{y} p_{2+}^{\prime} \rho_{*} \mathbf{D}_{z}(\text { see }[\operatorname{Vir} 04])\left(K_{\bullet}[-1]\right) \\
& \simeq \mathbf{D}_{y} p_{2+} \mathbf{D}_{z}\left(K_{\bullet}[-1]\right)
\end{aligned}
$$

Now we need the following statement

Lemma 3.3.2. There is a canonical isomorphism

$$
\mathcal{D}_{y \vee}^{\dagger}(\infty y \vee)[1] \simeq p_{2+} \mathbf{D}_{z}\left(K_{\bullet}\right)
$$

Proof. Using (8) , we know that $\mathcal{D}_{\mathrm{y}^{\vee} \leftarrow \mathcal{Z}}^{\dagger}(\infty) \otimes_{\mathcal{D}_{\mathcal{Z}}^{\dagger}(\infty)}^{\mathbf{L}} \mathbf{D}_{\mathcal{Z}}\left(K_{\bullet}\right)$ is represented by the following complex with terms in degrees -2 and -1

$$
\begin{gathered}
0 \longrightarrow \mathcal{D}_{y \vee \leftarrow z}^{\dagger}(\infty) \longrightarrow \mathcal{D}_{y \vee \leftarrow z}^{\dagger}(\infty) \longrightarrow 0 \\
P \longmapsto P\left(-\partial_{y}+\pi x\right) .
\end{gathered}
$$

Denote by $\mathcal{E}$ the -1 -cohomology group of this complex. Since the module $\mathcal{D}_{y^{\vee} \leftarrow z}^{\dagger}(\infty)$ is a right coherent $\mathcal{D}_{\mathcal{Z}}^{\dagger}(\infty)$-module, it is acyclic for the functor $\Gamma(\mathcal{Z},$.$) . It is also the case for \mathcal{E}$ 
by the long cohomology exact sequence and we can then compute $\Gamma(\mathcal{Z}, \mathcal{E})$ as the cokernel of the map

$$
\begin{gathered}
0 \longrightarrow \Gamma\left(z, \mathcal{D}_{y v_{\leftarrow} \leftarrow z}^{\dagger}(\infty)\right) \longrightarrow \Gamma\left(z, \mathcal{D}_{y \vee \leftarrow z}^{\dagger}(\infty)\right) \longrightarrow 0 \\
P \longmapsto P\left(-\partial_{y}+\pi x\right)
\end{gathered}
$$

In particular, we get an element $1 \in \Gamma(z, \mathcal{E})$, allowing us to consider a morphism $\varphi$ : $\mathcal{D}_{y \vee}^{\dagger}\left(\infty_{y \vee}\right) \rightarrow \mathbf{R} p_{2 *} \mathcal{E}$, that sends $P$ to $P \cdot 1$, where $1 \in R^{0} p_{2 *} \mathcal{E}$.

On the other hand, from the previous lemma 3.3.1, we know that $p_{2+} \mathbf{D}_{\mathcal{Z}}\left(K_{\bullet}[-1]\right)$, thus $\mathcal{E}$, is a coherent $\mathcal{D}_{y}^{\dagger}\left(\infty_{y}\right)$-module. By 1.5.2, it is enough to prove that the morphism induced on global sections of both sheaves is an isomorphism, to see that $\varphi$ is an isomorphism.

As $\mathcal{D}_{z}^{\dagger}(\infty)$-coherent module, $\mathcal{D}_{y \vee \leftarrow z}^{\dagger}(\infty)$ is acyclic for the functor $\Gamma$. Using the resolution given in 2.2, we identify $\Gamma\left(z, \mathcal{D}_{y^{\vee} \leftarrow z}^{\dagger}(\infty)\right)$ with $A_{2}(K)^{\dagger} / \partial_{x} A_{2}(K)^{\dagger}$. Finally, we get the following isomorphisms

$$
\begin{aligned}
\Gamma\left(z, \mathcal{D}_{y^{\vee} \leftarrow z}^{\dagger}(\infty)\right) / \Gamma\left(z, \mathcal{D}_{y^{\vee} \leftarrow z}^{\dagger}(\infty)\right)\left(-\partial_{y}+\pi x\right) & \simeq A_{2}(K)^{\dagger} / \partial_{x} A_{2}(K)^{\dagger}+A_{2}(K)^{\dagger}\left(-\partial_{y}+\pi x\right) \\
& \simeq B_{2}(K)^{\dagger} / \partial_{x} B_{2}(K)^{\dagger} \\
& \simeq A_{1}(K)^{\dagger} \\
& \simeq \Gamma\left(y^{\vee}, \mathcal{D}_{y \vee}^{\dagger}\left(\infty_{y \vee}\right)\right) .
\end{aligned}
$$

But $\mathbf{R} \Gamma(\mathcal{Z}, \mathcal{E})$ is isomorphic to $\Gamma(z, \mathcal{E})$ placed in degree 0 , and also to $\mathbf{R} \Gamma\left(\mathcal{Z}, \mathbf{R} p_{2 *} \mathcal{E}\right)$. Because the cohomology sheaves of $R p_{2 *} \mathcal{E}$ are acyclic for $\Gamma(y \vee,$.$) , the spectral sequence$ attached to composite functors $\Gamma\left(y^{\vee},.\right)$ and $p_{2 *}$ degenerates, proving that $R^{i} p_{2 *} \mathcal{E}=0$ for $i \neq 0$. Finally the previous computation gives that $p_{2 *} \mathcal{E}$, and thus $p_{2+} \mathbf{D}_{\mathcal{Z}}\left(K_{\bullet}\right)[-1]$ is isomorphic to $\mathcal{D}_{y v}^{\dagger}\left(\infty_{y v}\right)$ (in degree 0 ) and the lemma.

We finally get

$$
\begin{aligned}
\mathcal{F}_{!}\left(\mathcal{D}_{y}^{\dagger}\left(\infty_{y}\right)\right) & \simeq \mathbf{D}_{y} p_{2+} \mathbf{D}_{z}\left(K_{\bullet}[-1]\right) \\
& \simeq \mathbf{D}_{y}\left(\mathcal{D}_{y \vee}^{\dagger}\left(\infty_{y \vee}\right)[2]\right) \\
& \simeq \mathcal{D}_{y \vee}^{\dagger}\left(\infty_{y \vee}\right)[-1] \\
& \simeq \mathcal{F}\left(\mathcal{D}_{y}^{\dagger}\left(\infty_{y}\right)\right) 2.3 .2,
\end{aligned}
$$

It remains to show that the canonical map $\mathcal{F}_{!}\left(\mathcal{D}_{y}^{\dagger}\left(\infty_{y}\right)\right) \rightarrow \mathcal{F}\left(\mathcal{D}_{y}^{\dagger}\left(\infty_{y}\right)\right)$ maps 1 to 1 . For this we observe that the canonical map

$$
p_{2+}^{\prime} \mathbf{D}_{z}^{\prime} \rho_{*} \mathbf{D}_{\mathcal{Z}}\left(K_{\bullet}\right)[-1] \rightarrow p_{2+} \mathbf{D}_{z} \mathbf{D}_{\mathcal{Z}}\left(K_{\bullet}\right)[-1]
$$


maps 1 (considered as an element of the 0-cohomology group of these complexes) to 1 , which is clear from the explicit computation of the complex $K_{\text {. }}$ and the map of functors $p_{2+}^{\prime} \mathbf{D}_{z}^{\prime} \rightarrow p_{2+} \mathbf{D}_{z}$. Then identification of $\mathbf{D}_{z} \mathbf{D}_{\mathcal{Z}}\left(K_{\bullet}\right)$ also maps 1 to 1 , and this finally gives us the isomorphism

$$
\mathcal{F}_{!}\left(\mathcal{D}_{y}^{\dagger}(\infty y)\right) \simeq \mathcal{F}\left(\mathcal{D}_{y}^{\dagger}(\infty y)\right)
$$

\section{References}

[AM11] Tomoyuki Abe and Adriano Marmora. Product formula for $p$-adic epsilon factors. arxiv, pages 1-77, 2011.

[Ber96] P. Berthelot. D-modules arithmétiques I. Opérateurs différentiels de niveau fini. Ann. scient. Éc. Norm. Sup., $4^{e}$ série, t. 29, p.185-272, 1996.

[Ber00] P. Berthelot. D-modules arithmétiques II descente par Frobenius. Bull. Soc. Math. France, Mémoire 81, p. 1-135, 2000.

[Huy95a] C. Huyghe. Construction et étude de la Transformation de Fourier pour les D-modules arithmétiques. Thèse de Doctorat, Université de Rennes I, 1995.

[Huy95b] C. Huyghe. Interprétation géométrique sur l'espace projectif des $A_{N}(K)^{\dagger}$-modules cohérents. C. R. Acad. Sci. Paris, t. 321, Série I, p. 587-590, 1995.

[Huy98] C. Huyghe. $D^{\dagger}$-affinité des schémas projectifs. Ann. Inst. Fourier, t. 48, fascicule 4, p. 913-956, 1998.

[Huy03] C. Huyghe. Un théorème de comparaison entre les faisceaux d'opérateurs différentiels de Berthelot et de Mebkhout-Narvaez-Macarro. Journal of Algebraic Geometry, 12, No. 1, p. 147-199, 2003.

[KL85] N. Katz and G. Laumon. Transformation de Fourier et majoration de sommes exponentielles. Publ. Math. I.H.E.S., 62, p. 361-418, 1985.

[NH04] Christine Noot-Huyghe. Transformation de Fourier des $\mathcal{D}$-modules arithmétiques. I. In Geometric aspects of Dwork theory. Vol. I, II, pages 857-907. Walter de Gruyter GmbH \& Co. KG, Berlin, 2004. 
[NH07] C. Noot-Huyghe. Finitude de la dimension homologique d'algèbres d'opérateurs différentiels faiblement complètes et à coefficients surconvergents. J. Algebra, $307(2), 2007$.

[Vir00] A. Virrion. Dualité locale et holonomie pour les D-modules arithmétiques. Bull. Soc. Math. France, t. 321, p. 101-168, 2000.

[Vir04] Anne Virrion. Trace et dualité relative pour les D-modules arithmétiques. In Geometric aspects of Dwork theory. Vol. I, II, pages 1039-1112. Walter de Gruyter GmbH \& Co. KG, Berlin, 2004.

Christine Huyghe

IRMA, UFR de mathématiques

Université Louis Pasteur

7, rue René Descartes

67084 STRASBOURG cedex FRANCE

mél huyghe@math.unistra.fr, http://www-irma.u-strasbg.fr/ huyghe 